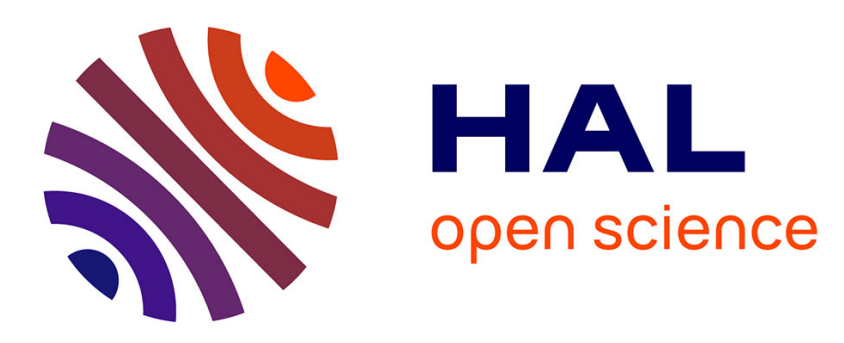

\title{
Biased Spin-State Energetics of Fe(II) Molecular Complexes within Density-Functional Theory and the Linear-Response Hubbard U Correction
}

Lorenzo Antonio Mariano, Bess Vlaisavljevich, Roberta Poloni

\section{- To cite this version:}

Lorenzo Antonio Mariano, Bess Vlaisavljevich, Roberta Poloni. Biased Spin-State Energetics of Fe(II) Molecular Complexes within Density-Functional Theory and the Linear-Response Hubbard U Correction. Journal of Chemical Theory and Computation, 2020, 16 (11), pp.6755-6762. 10.1021/acs.jctc.0c00628 . hal-03046264

\section{HAL Id: hal-03046264 https://hal.science/hal-03046264}

Submitted on 8 Dec 2020

HAL is a multi-disciplinary open access archive for the deposit and dissemination of scientific research documents, whether they are published or not. The documents may come from teaching and research institutions in France or abroad, or from public or private research centers.
L'archive ouverte pluridisciplinaire $\mathbf{H A L}$, est destinée au dépôt et à la diffusion de documents scientifiques de niveau recherche, publiés ou non, émanant des établissements d'enseignement et de recherche français ou étrangers, des laboratoires publics ou privés. 


\section{Biased spin-state energetics of $\mathrm{Fe}$ (II) molecular complexes within density functional theory and the linear-response Hubbard- $U$ correction Lorenzo A. Mariano, ${ }^{\dagger}$ Bess Vlaisavljevich, ${ }^{\ddagger}$ and Roberta Poloni* ${ }^{* \dagger}$ $\dagger$ Grenoble-INP, SIMaP, University of Grenoble-Alpes, CNRS, F-38042 Grenoble, France $\ddagger$ Department of Chemistry, University of South Dakota, Vermillion, South Dakota 57069, USA}

Received October 16, 2020; E-mail: roberta.poloni@grenoble-inp.fr

\begin{abstract}
The spin-state energetics of six $\mathrm{Fe}(\mathrm{II})$ molecular complexes are computed using the linear-response Hubbard $U$ approach within DFT. The adiabatic energy differences, $\Delta E_{\mathrm{H}-\mathrm{L}}$, between the high spin $(\mathrm{S}=2)$ and the low spin $(\mathrm{S}=0)$ states are computed and compared with accurate coupled clustercorrected CASPT2 results. We show that DFT+U fails in correctly capturing the ground state for strong field-ligands yielding $\Delta E_{\mathrm{H}-\mathrm{L}}$ that are almost constant throughout the molecular series. This bias towards high spin together with the metal/ligand charge transfer upon $U$ correction are here quantified and explained using molecular orbital diagrams involving both $\sigma$ - and $\pi$-bonding interactions. With increasing ligandfield strengths this bias also increases owing to the stronger molecular character of the metal/ligand Kohn-Sham orbitals thus resulting in large deviations from the reference larger than $4 \mathrm{eV}$. Smaller values of $U$ can be employed to mitigate this effect and recover the right energetics.
\end{abstract}

The past decade has seen a substantial effort from the computational chemistry community in predicting the spin-state energetics of molecular complexes containing transition-metal atoms. ${ }^{1-8}$ Achieving such a description with chemical accuracy appears today as a significant challenge for ab initio methods with strong implications in future assistance and guidance of experimental efforts devoted to the (i) development and optimization of catalytic reactivity and the (ii) design of novel spin crossover (SCO) complexes. The vast majority of SCO materials, which are of great interest for applications such as molecular spintronics, molecular electronics, sensors and actuators, ${ }^{9-12}$ are octahedrallycoordinated $\mathrm{Fe}(\mathrm{II})$-based molecular complexes. For these complexes, the thermodynamics of the spin transition (i.e. the transition temperature $\mathrm{T}_{1 / 2}$ ) between the low-spin (LS) and high-spin (HS) states is dominated by the adiabatic energy difference, $\Delta E_{\mathrm{H}-\mathrm{L}}=E_{\mathrm{HS}}-E_{\mathrm{LS}}$, i.e. the energy difference between the two spin states computed at their corresponding geometry. Within density functional theory, large deviations in the value of $\Delta E_{\mathrm{H}-\mathrm{L}}$ are found among different families of exchange and correlation functionals. ${ }^{1,5,6,13-15}$ Semilocal functionals, such as GGA for example, overstabilize LS, ${ }^{13,16-20}$ while Hartree-Fock overstabilizes HS. ${ }^{21}$ Thus, depending on the system of choice, accurate $\Delta E_{\mathrm{H}-\mathrm{L}}$ may be obtained by tuning the amount of exact exchange in global hybrids, ${ }^{21,22}$ or by adoping density-corrected approaches. ${ }^{15}$

Since its original formulation, ${ }^{23,24}$ the capability of Hubbard $U$-corrected $\mathrm{DFT}^{25,26}$ in solving the deficiencies of standard semilocal functionals, mostly owing to self-interaction error, ${ }^{27-30}$ has allowed it to provide a quantitatively correct description of varying properties of transition metal complexes. ${ }^{31-34}$ Its performance in predicting spin-state energetics has also been studied, to some extent. DFT $+\mathrm{U}$ with a self consistent $U$ averaged among different spin states was shown to significantly improve upon DFT. ${ }^{35,36}$ In Ref. 37 , by using a $U$ computed at different spin-state configurations the authors correctly predicted the ground state and the experimentally-observed pressure-induced SCO for $(\mathrm{Mg}, \mathrm{Fe})(\mathrm{Si}, \mathrm{Fe}) \mathrm{O}_{3}$ perovskites. Several studies have reported quantitative agreement with experiment by adopting a value of $U$ of $4 \mathrm{eV} ;^{38-40}$ other studies, instead, show the necessity to parametrize $U$ against experiment for a quantitative description of the energetics. ${ }^{41-44}$ Vela and coworkers recently showed that the linear response $U$-approach yields an adiabatic energy difference for a well-known SCO molecular crystal, Fe(phen $)_{2}(\mathrm{NCS})_{2}$, deviating almost $1.8 \mathrm{eV}$ from the experimentally-extracted reference value. ${ }^{44}$

Here, we report a comprehensive analysis of the performance of the $\mathrm{DFT}+\mathrm{U}$ method to predict adiabatic energy differences of 6 -fold-coordinated $\mathrm{Fe}(\mathrm{II})$ complexes and provide an explanation of the apparent contrasting findings mentioned above, thus clarifying the limitations of this approach.

We compute the $\Delta E_{\mathrm{H}-\mathrm{L}}$ between the ${ }^{1} A_{1 g}$ LS and the ${ }^{5} T_{2 g}$ HS states of six $\mathrm{Fe}(\mathrm{II})$ octahedral complexes with varying ligand-field strengths by employing LDA, GGA and their Hubbard $U$-corrected version. Specifically, we use LDA, $\mathrm{LDA}+\mathrm{U}_{\mathrm{sc}}, \mathrm{PBE}$ and $\mathrm{PBE}+\mathrm{U}_{\mathrm{sc}}$, where the linear responseHubbard $U,{ }^{45}$ named $U_{\mathrm{sc}}$, is computed for each spin state. We then report $\Delta E_{\mathrm{H}-\mathrm{L}}$ computed with a $\mathrm{PBE}+\mathrm{U}$ approach where the same $U$ is used for both LS and HS, in the same spirit of previous studies. ${ }^{35,36,38-44}$ As a reference method for $\Delta E_{\mathrm{H}-\mathrm{L}}$ we compute coupled cluster-corrected CASPT2 energies, i.e. CASPT2/CC, a method recently proposed by Pierloot and coworkers. ${ }^{46,47}$ Such an approach allows for the improvement of the description of electronic correlations in semicore 3s3p electrons by adopting $\operatorname{CCSD}(\mathrm{T})$ thus removing the well-known bias of CASPT2 towards high-spin states. $^{46,47}$

We show a systematic overstabilization of HS states by $\mathrm{DFT}+\mathrm{U}_{\mathrm{sc}}$ with deviations from the reference CASPT2/CC values reaching up to $4.5 \mathrm{eV}$ for strong-field ligands. Such an overstabilization arises from the penalizing Hubbard energy term in the total energy which is systematically larger in the LS state compared to HS. This effect has two origins: both the summation term that multiplies $U$ in the Hubbard energy term (vide infra) and $U_{\text {sc }}$ are always larger in LS. We here explain in detail the origin of the first one. We show that this failure of $\mathrm{DFT}+\mathrm{U}_{\mathrm{sc}}$ can be mitigated by empirically choosing $U$ values that reduce this bias. The density change upon application of $U$ is here rationalized in terms of the change in metal/ligand hybridization effectively resulting in a metal-ligand charge transfer whose extent and sign depends on both the spin state and the ligand-field strength. 

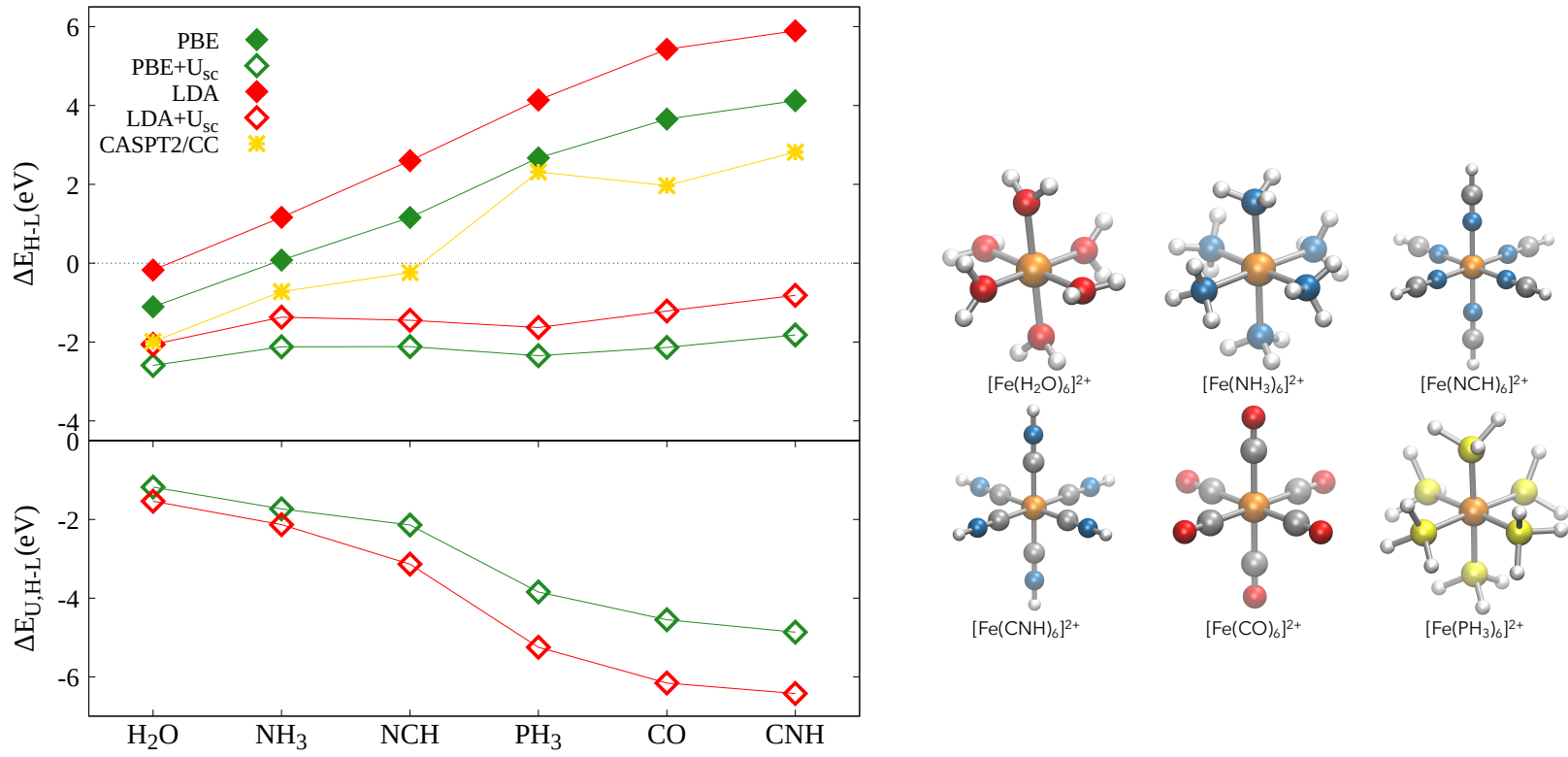

Figure 1. Adiabatic energy differences, $\Delta E_{\mathrm{H}-\mathrm{L}}$, computed with different approaches and the difference between the $\mathrm{E}_{\mathrm{U}}$ term of LS and $\mathrm{HS}$ computed using $\mathrm{PBE}+\mathrm{U}_{\mathrm{sc}}$ (left panels). The $\mathrm{CASPT} / \mathrm{CC}$ reference values are $-1.99 \mathrm{eV},-0.72 \mathrm{eV},-0.24 \mathrm{eV}, 2.31 \mathrm{eV}, 1.97 \mathrm{eV}$ and $2.82 \mathrm{eV}$, from weak to strong-field ligands. Molecular complexes studied in this work (right figure).

The term hybridization is here employed to describe mixing of metal and ligand atomic orbitals.

Recall that the Hubbard $U$ term enters a DFT calculation as an extra potential acting self-consistently on the Kohn-Sham wave functions $\left|\Psi_{i}^{\sigma}\right\rangle$ of orbital index i and spin $\sigma$ :

$$
V_{\mathrm{DFT}+\mathrm{U}}=V_{\mathrm{DFT}}\left|\Psi_{i}^{\sigma}\right\rangle+\sum_{m} U\left(\frac{1}{2}-n_{m}^{\sigma}\right)\left|\phi_{m}\right\rangle\left\langle\phi_{m} \mid \Psi_{i}^{\sigma}\right\rangle
$$

where the projectors $\left\{\left|\phi_{m}\right\rangle\right\}$ are here atomic-like functions (the five $3 \mathrm{~d}$ atomic functions) and the occupations, $\left\{n_{m}^{\sigma}\right\}$, are the eigenvalues of the $5 \times 5$ occupation matrix $n_{m m^{\prime}}^{\sigma}=$ $\sum_{i} f_{i}^{\sigma}\left\langle\Psi_{i}^{\sigma} \mid \phi_{m}\right\rangle\left\langle\phi_{m^{\prime}} \mid \Psi_{i}^{\sigma}\right\rangle$, with $f_{i}^{\sigma}$ being Fermi-Dirac occupations. Equation 1 shows that the Hubbard potential is repulsive for $n<1 / 2$, attractive for $n>1 / 2$ and zero for $n=1 / 2$. The $\mathrm{DFT}+\mathrm{U}$ energy functional reads:

$$
E_{\mathrm{DFT}+\mathrm{U}}\left[\rho(\mathbf{r}),\left\{n_{m}^{\sigma}\right\}\right]=E_{\mathrm{DFT}}[\rho(\mathbf{r})]+\sum_{m, \sigma} \frac{U}{2}\left[n_{m}^{\sigma}\left(1-n_{m}^{\sigma}\right)\right]
$$

For each molecule at either spin state the following strategy is adopted: (1) $U$ is first computed with linear response $^{45}$ by employing a DFT (without $U$ ) optimized geometry; then (2) this $U$ is used to optimize again the geometry and (3) another $U$ is calculated on the new structure. This process is iterated until convergence is reached (see SI for more details). This self-consistent and structurally-consistent $U$ is named $U_{\mathrm{sc}}{ }^{48}$ This procedure is performed using both LDA and PBE by employing QUANTUM EsPREsso. ${ }^{49}$ The reference coupled cluster-corrected CASPT2 adiabatic energy differences, $\Delta E_{\mathrm{CASPT} 2 / \mathrm{CC}}$, are computed as follows, by adopting the procedure of Pierloot and coworkers: ${ }^{47}$

$$
\begin{gathered}
\Delta E_{\mathrm{CASPT} 2 / \mathrm{CC}}=\Delta E_{\mathrm{CASPT} 2}+\Delta E_{3 \mathrm{~s} 3 \mathrm{p}, \mathrm{CCSD}(\mathrm{T})} \\
\Delta E_{3 \mathrm{~s} 3 \mathrm{p}, \mathrm{CCSD}(\mathrm{T})}=\Delta E_{+3 \mathrm{~s} 3 \mathrm{p}}-\Delta E_{\mathrm{no}-3 \mathrm{~s} 3 \mathrm{p}}
\end{gathered}
$$

Extended multi-state (XMS) CASPT2 calculations were performed using BAGEL ${ }^{50,51}$ on the TPSSH $^{52,53}$-optimized geometries for both HS and LS by using aug-cc-pVQZ-DK basis sets and an active space of 10 electrons on 12 orbitals, (10e,12o). This includes the 3d electrons of Fe(II), the two ligand $-e_{g}$ molecular orbitals plus the Fe 4 d doubleshell, ${ }^{1,5}$ and their corresponding electrons (Figure S1 and S2). The CASPT2 calculations used for the reference set were performed without any ionisation potential-electron affinity (IPEA) shift to the zeroth-order Hamiltonian. Because this does affect energy differences of different spin states, ${ }^{46,54}$ we report also the $\Delta E_{\mathrm{CASPT} 2}$ computed with an IPEA $=0.25$ a.u. in Table S3. For $\left[\mathrm{Fe}\left(\mathrm{H}_{2} \mathrm{O}\right)_{6}\right]^{+2}$ we were unable to converge a $(10 \mathrm{e}, 12 \mathrm{o})$ active space where the two ligand $-e_{g}$ orbitals remained in the active space for LS. The Fe 3 s orbital consistently rotated into the active space replacing one of the ligand $e_{g}$ orbitals. Thus, for water the $\Delta E_{\mathrm{CASPT} 2}$ is taken from $(10 \mathrm{e}, 12 \mathrm{o})$ calculations by Gagliardi and coworkers. ${ }^{1}$ See computational methods in the SI for more details. To add the metal semicore $3 \mathrm{~s} 3 \mathrm{p}$ correlations, $\Delta E_{3 \mathrm{~s} 3 \mathrm{p}, \mathrm{CCSD}(\mathrm{T})}$, to the CASPT2 energy difference, $\operatorname{CCSD}(\mathrm{T})$ was performed using ORCA ${ }^{55,56}$ on the same TPSSH-optimized geometries by including and freezing the 3s3p electrons ${ }^{47}$ (equation 4). The aug-cc-pwCVTZ-DK and cc-pVDZ basis sets were used for $\mathrm{Fe}$ and the ligand atoms, respectively. The whole set of CASPT2, 3s $3 p$ contribution and final CASPT2/CC adiabatic energy differences is reported in Table S1. CASPT2 values obtained with an active space of $(6 \mathrm{e}, 10 \mathrm{o})$ and the ANO-RCC basis sets are also reported for comparison in Table $\mathrm{S} 2$.

We note that accurate adiabatic energy differences from diffusion Monte Carlo (DMC) are also available in the literature for $\mathrm{Fe}(\mathrm{NCH})_{6}^{2+} .{ }^{57}$ By adopting different choices of the trial wavefunction and including a multireference treatment, the authors report a best estimate value that varies between -0.55 and $-0.95 \mathrm{eV}$, depending on the geometry. Our CASPT2/CC calculations yield $-0.24 \mathrm{eV}$. For $\mathrm{Fe}\left(\mathrm{NH}_{3}\right)_{6}^{2+}$ and $\mathrm{Fe}\left(\mathrm{H}_{2} \mathrm{O}\right)_{6}^{2+}$, Ref. 15 reports DMC energy differences of -1.23 $\mathrm{eV}$ and $-1.78 \mathrm{eV}$, respectively. We find $-0.72 \mathrm{eV}$ and $-1.99 \mathrm{eV}$. 

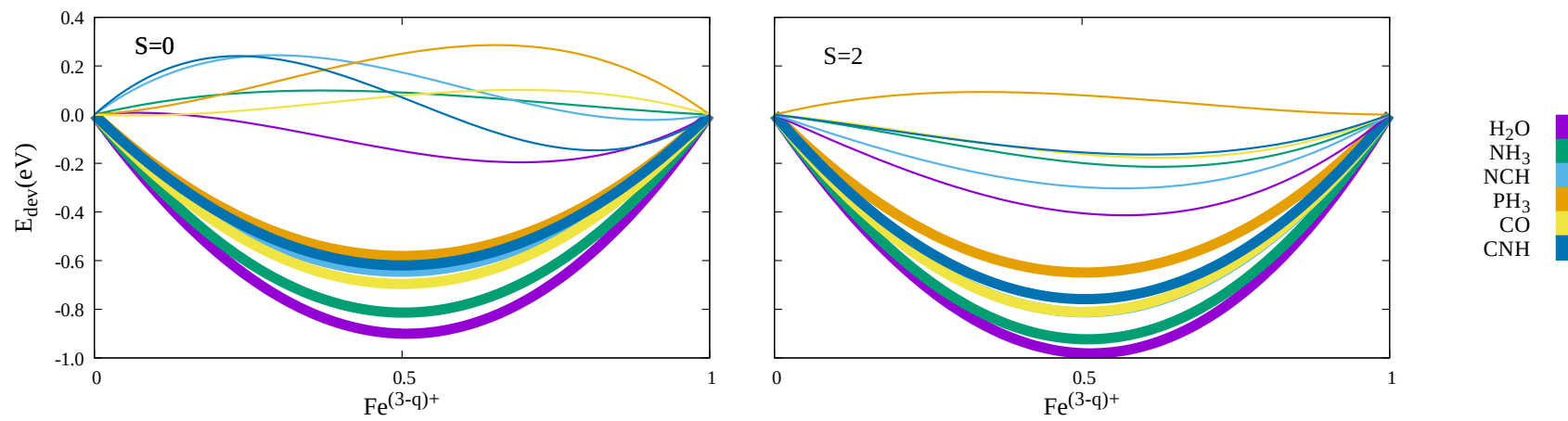

Figure 2. Deviation from linearity of the total energy with respect to the total charge of the system, from $\mathrm{Fe}^{3+}$ to $\mathrm{Fe}^{2+}$, for the six molecular complexes, computed using PBE. A lower deviation is found when the Hubbard- $U$ approach is employed. Thick and thin lines refer to $\mathrm{DFT}$ and $\mathrm{DFT}+\mathrm{U}_{\mathrm{sc}}$ results.

The non-negligible difference between the two approaches reflects the limitations associated to each method, mainly the choice of the active space and that of the trial wavefunction. A significantly larger discrepancy, possibly due to the multiconfigurational character of $\mathrm{LS}$, is found for $\mathrm{Fe}(\mathrm{CO})_{6}^{2+}$, whose $\Delta E_{\mathrm{H}-\mathrm{L}}$ is reported to be $+0.59 \mathrm{eV}$ in Ref. 15 using a single-determinant trial wavefunction. We compute a value of $+1.97 \mathrm{eV}$ using CASPT2/CC.

The DFT and DFT $+\mathrm{U}$ results for $\Delta E_{\mathrm{H}-\mathrm{L}}$ are shown in Figure 1 (Tables S5-S6) for increasing ligand field strengths together with our CASPT2/CC calculations. LDA and PBE systematically stabilize LS states possibly due to the wellknown delocalization error. ${ }^{13,17-20,58}$ LDA shows an even larger stabilization of LS possibly due to greater electronic delocalization as compared to PBE. ${ }^{59}$ Conversely, DFT $+\mathrm{U}_{\mathrm{sc}}$ overstabilizes HS with an increasing deviation from reference CASPT2/CC results for stronger-field ligands. Remarkably, for weak-field ligands (i.e. for $\Delta E_{\mathrm{CASPT} 2 / \mathrm{CC}}<0$ ), among $\mathrm{PBE}, \mathrm{PBE}+\mathrm{U}_{\mathrm{sc}}, \mathrm{LDA}$ and $\mathrm{LDA}+\mathrm{U}_{\mathrm{sc}}$, the best agreement with the reference calculations is found for $\mathrm{LDA}+\mathrm{U}_{\mathrm{sc}}$, (see in Table 1), with a mean absolute error (MAE) of 0.644 $\mathrm{eV}$, in agreement with findings by Zhang and coworkers. ${ }^{39}$ As we demonstrate below, this agreement is fortuitous and results from a cancellation of errors: the LS overstabilization by LDA is compensated for by the HS overstabilitation by the $U$-corrected LDA. For strong-field ligands (i.e. for $\left.\Delta E_{\mathrm{CASPT} 2 / \mathrm{CC}}>0\right)$ the best agreement is achieved with $\mathrm{PBE}$ $(\mathrm{MAE}=1.115 \mathrm{eV})$ and the deviation of $\mathrm{DFT}+\mathrm{U}_{\mathrm{sc}}$ from the reference CASPT2/CC increases up to $4.5 \mathrm{eV}$ (see Figure 1 and Table 1).

Table 1. Mean absolute error (MAE) in eV for weak-field, strongfield ligand molecules, and the total value.

\begin{tabular}{|c|l|l|l|l|}
\hline \hline \multicolumn{5}{|c|}{ MAE } \\
\hline Functional & $U$ & weak-field & strong-field & Total \\
\hline \multirow{5}{*}{ PBE } & 0 & 1.026 & 1.115 & 1.071 \\
& 2 & 0.376 & 0.354 & 0.365 \\
& 4 & 0.300 & 1.315 & 0.808 \\
& 6 & 0.807 & 2.382 & 1.595 \\
& 8 & 1.281 & 3.535 & 2.408 \\
& $U_{\text {sc }}$ & 1.291 & 4.462 & 2.876 \\
\hline \multirow{2}{*}{ LDA } & 0 & 2.179 & 2.785 & 2.482 \\
& $U_{\text {sc }}$ & 0.644 & 3.582 & 2.113 \\
\hline
\end{tabular}

We note that $\mathrm{PBE}+\mathrm{U}_{\mathrm{sc}}$ performs worse than PBE despite that the $U_{\mathrm{sc}}$ approach leads to a better electronic density with reduced self-interaction error. ${ }^{45,60}$ This is shown in Figure 2 where the deviation from linearity of the total energy with respect to fractional occupancy, which arises from the self-interaction error, ${ }^{28,29,61,62}$ is reported for the six molecular complexes, for $\mathrm{PBE}$ and $\mathrm{PBE}+\mathrm{U}_{\mathrm{sc}}$. Kronik and Baer demonstrated that the average deviation from linearity is given by the energy difference between the LUMO of $\mathrm{N}$-electron system and the HOMO of the $\mathrm{N}+1$-electron system. ${ }^{63}$ The deviation as a function of the fractional charge, $\mathrm{q}$, is computed as the difference between the energy $E(\mathrm{q})$ and the linear interpolation between the $\mathrm{q}=0 \mathrm{LUMO}$ and the $\mathrm{q}=1$ HOMO, which correspond to the $\mathrm{N}$ and $\mathrm{N}+1$-electron system, here $\mathrm{Fe}^{3+}$ and $\mathrm{Fe}^{2+}$, respectively. $E(\mathrm{q})$ is computed by interpolating $E(\mathrm{q}=0)$ and $E(\mathrm{q}=1)$ using a cubic function which depends through $q$ upon the total energy difference $\Delta E_{\mathrm{q}}=E(\mathrm{q}=0)-E(\mathrm{q}=1)$, and the $\mathrm{q}=1 \operatorname{HOMO}\left(\mathrm{Fe}^{2+}\right)$, $\epsilon_{q=1}^{H O M O}$, and $\mathrm{q}=0 \mathrm{LUMO}\left(\mathrm{Fe}^{3+}\right), \epsilon_{q=0}^{L U M O}$, eigenvalues: ${ }^{64,65}$

$$
\begin{aligned}
E_{\mathrm{dev}}(q)= & {\left[\left(\epsilon_{q=0}^{\mathrm{LUMO}}-\Delta E_{q}\right)(1-q)+\right.} \\
& \left.+\left(\Delta E_{q}-\epsilon_{q=1}^{\mathrm{HOMO}}\right) q\right] q(1-q) .
\end{aligned}
$$

This function approximates the energy versus fractional occupancy curve without the need for explicit fractional-charge calculations. ${ }^{64}$ Figure 2 shows that, overall, DFT $+\mathrm{U}_{\mathrm{sc}}$ decreases the SIE, as demonstrated by the systematic decrease of $E_{\mathrm{dev}}$, upon $U$ correction, for both LS and HS. Thus, while the density overall improves $U$, the $\mathrm{DFT}+U$ energetics do not: this is because the functional form of the Hubbard energy within the DFT $+\mathrm{U}$ approach, $E_{\mathrm{U}}$, leads to a bias toward the high-spin state due to the larger penalization of LS than HS. ${ }^{66,67}$ Such a penalization arises from two effects: the larger values of both $U_{\mathrm{sc}}$ and the summation term, $\sum_{m, \sigma}\left[n_{m}^{\sigma}\left(1-n_{m}^{\sigma}\right)\right]$, in LS. The Hubbard- $U$ energy term that enters the total energy in equation (2), is the largest (destabilizing) when the total summation for a given $U$ is the biggest, which is true when the $\left\{n_{m}^{\sigma}\right\}$ are close to $1 / 2$.

A few important aspects should be commented on at this point to understand the results in Figure 1. These are summarized below and shown in Figure 3 where the summation, $\sum_{m, \sigma}\left[n_{m}^{\sigma}\left(1-n_{m}^{\sigma}\right)\right]$, is plotted separately from the occupations of the $e_{g}$ and the $t_{2 g}$ states: (1) the summation term increases for increasing ligand-field strengths as a result of the larger $\mathrm{M} / \mathrm{L}$ hybridization; ${ }^{20}$ (2) this term is larger for LS than HS, as already pointed out; ${ }^{66}$ (3) the difference in this term between LS and HS increases for strong-field ligands (see Figure 3); (4) the difference between the $U_{\mathrm{sc}}$ of LS and HS also increases as a function of the ligand field 

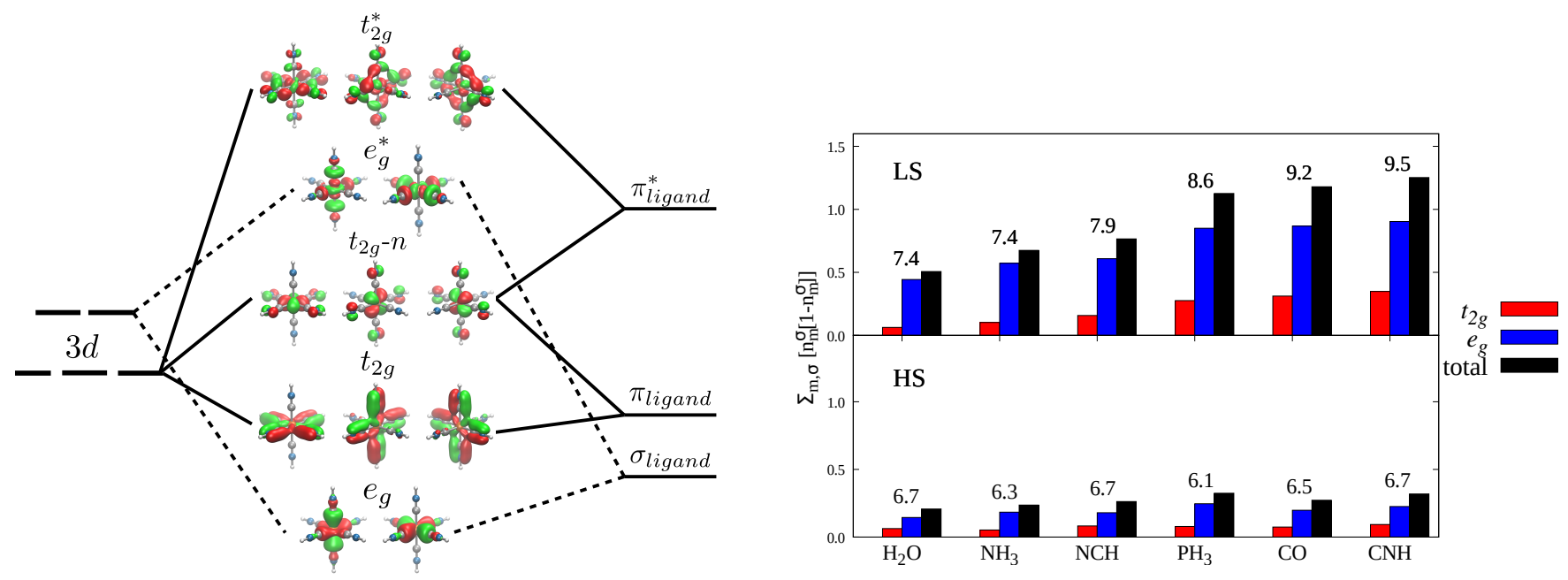

Figure 3. Left panel: molecular-orbital diagram built from both $\sigma$ and $\pi$-bonding together with the corresponding Kohn-Sham orbitals for $\mathrm{Fe}(\mathrm{CNH})_{6}^{2+}$. Right panel: the $\sum_{m, \sigma}\left[n_{m}^{\sigma}\left(1-n_{m}^{\sigma}\right)\right]$ term is plotted for the whole molecular series for increasing strength of the ligand-field and separately for each spin states, $\sigma$, and orbital symmetry. The $U$ values $(\mathrm{eV})$ are also reported.

strength. Hybridization is here quantified by performing the Hirshfeld polulation analysis in order to decompose the active molecular orbitals from the CASSCF calculation into their corresponding atomic compositions (Tables S7-S11). These trends yield a negative difference of the $U$ energy term between HS and LS, i.e. $\Delta E_{\mathrm{U}, \mathrm{H}-\mathrm{L}}=E_{\mathrm{U}}{ }^{\mathrm{HS}}-E_{\mathrm{U}}{ }^{\mathrm{LS}}$, that largely increases as a function of the ligand-field strength, as shown in the lower panels of Figure 1. This explains the increasing bias towards HS states for ligands on the right side of the spectrochemical series. As a consequence, the $\mathrm{DFT}+\mathrm{U}$-computed adiabatic energy differences are always negative and their dispersion across the series no longer reflects the trend expected for varying ligand field strengths as predicted by CASPT2/CC. While PBE and LDA do follow the CASPT2/CC behavior, the Hubbard- $U$ corrected calculations show no correlation with the reference set.

In what follows we explain precisely the origin of the systematically larger summation term for LS than HS, i.e. why the occupations $\left\{n_{m}^{\sigma}\right\}$ associated to both the $t_{2 g^{-}}$and $e_{g^{-}}$ like states are systematically closer to $1 / 2$. Since the $\left\{n_{m}^{\sigma}\right\}$ occupation numbers results from the projection of the occupied Kohn-Sham states, only, onto the atomic basis $\left\{\left|\phi_{m}\right\rangle\right\}$, fractional values of the $\left\{n_{m}^{\sigma}\right\}$ occurr when (1) the d orbitals of Fe hybridize with the ligand and (2) the resulting $\mathrm{M} / \mathrm{L}$ hybridized molecular orbitals are only partially occupied. When all of these molecular orbitals are occupied, even though there is strong hybridization, the $\left\{n_{m}^{\sigma}\right\}$ are close to 1 (and zero when empty) thus yielding a low $\sum_{m, \sigma}\left[n_{m}^{\sigma}\left(1-n_{m}^{\sigma}\right)\right]$.

As illustrated in the left panel of Figure 3, the two sets of molecular orbitals arising from the hybridization of the metal and ligand via the $\sigma$-bond are the bonding $e_{g}$ and the antibonding $e_{g}^{*}$. For LS states, the bonding (ligand) $e_{g}$ are occupied while the $e_{g}^{*}$ states, with larger metal-like character, are empty (see also projected density of states in Figures S4-S15). Thus, fractional $\left\{n_{m}^{\sigma}\right\}$ arise from the projection of these occupied ligand $e_{g}$ onto the atomic basis because these states do exhibit some metal-like character. For HS states, the $e_{g}^{*}$ are empty only for one spin channel, yielding to a total summation term that is lower than in LS, for which both spin channels are summed up in the summation.
Additionally, the larger bond lengths in the HS geometries result from occupied antibonding states that yield a smaller hybridization. This, as it is well known, contributes to a further decrease in the fractional occupation and is supported by the Hirshfeld population analysis (Tables S7-S11).

Similar arguments hold to explain the larger fractional occupations of the $t_{2 g}$ states in LS than in HS. The hybridization between the metal non-bonding orbitals and the ligand states via $\pi$-bonding gives rise to three sets of molecular orbitals $^{68}$ which exhibit $\pi$ bonding, non-bonding and antibonding character for increasing energy, and are here referred to as $t_{2 g}, t_{2 g}-\mathrm{n}$ and $t_{2 g}^{*}$, respectively (Figure 3 and Figures S4-S15). Overall, the $\sum_{m, \sigma}\left[n_{m}^{\sigma}\left(1-n_{m}^{\sigma}\right)\right]$ associated to the $t_{2 g}$ set is significantly smaller than for the $e_{g}$ set as expected from the smaller hybridization resulting from a $\pi$ bond versus a $\sigma$-bond (Tables S7-S11 and Figures S4-S15).

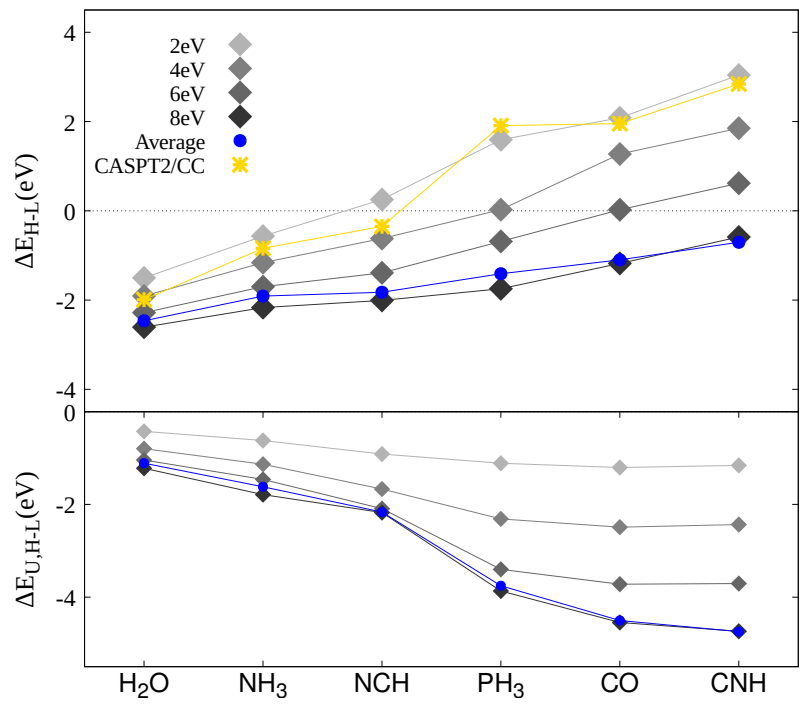

Figure 4. Adiabatic energy differences, $\Delta E_{\mathrm{H}-\mathrm{L}}$, (upper panel) and energy difference of the Hubbard term, $\Delta E_{\mathrm{U}, \mathrm{H}-\mathrm{L}}$, computed at different values of $U$, by using PBE.

The correlation between ligand-field strength and 
$\sum_{m, \sigma}\left[n_{m}^{\sigma}\left(1-n_{m}^{\sigma}\right)\right]$ follows from the larger hybridization of the molecular orbitals for stronger-field ligands.

$\mathrm{LDA}+\mathrm{U}_{\mathrm{sc}}$ gives the same qualitative trend across the molecular series as $\mathrm{PBE}+\mathrm{U}_{\mathrm{sc}}$ but with a larger deviation from the LDA result (Figure 1). The $\Delta E_{\mathrm{U}, \mathrm{H}-\mathrm{L}}$ increases more in LDA than in PBE across the molecular series due to the larger values of $U_{\mathrm{sc}}$ (Table S5) and larger fractional values for occupations $\left\{n_{m}^{\sigma}\right\}$ computed in LS using LDA as compared to PBE (Figure S3). The better performance of $\mathrm{LDA}+\mathrm{U}$ compared to $\mathrm{PBE}+\mathrm{U}$, claimed previously for weak ligands ${ }^{39}$ may now be understood: the bias introduced by $E_{\mathrm{U}}$ rigidly downshifts in energy the LDA- and PBE-computed $\Delta E_{\mathrm{H}-\mathrm{L}}$. In the case of $\left[\mathrm{Fe}\left(\mathrm{H}_{2} \mathrm{O}\right)_{6}\right]^{2+}$, the overstabilization of LS by LDA (approx. $2 \mathrm{eV}$ ) and the bias introduced by $\mathrm{LDA}+\mathrm{U}$ cancel out yielding a $\Delta E_{\mathrm{H}-\mathrm{L}}$ in remarkably good agreement with CASPT2/CC data, with a deviation smaller than 0.1 $\mathrm{eV}$.

The larger values of $U_{\mathrm{sc}}$ computed for LS compared to HS may derive from the overestimation of $U_{\mathrm{sc}}$ for LS as already discussed in the literature. ${ }^{38,69,70}$ Such an overestimation is here reflected by the overcorrection of the density produced by $U_{\mathrm{sc}}$ in LS and is shown by the concave $\mathrm{E}_{\mathrm{dev}}$ in Figure 2 . From the above observations, since the penalizing Hubbard term, $\mathrm{E}_{\mathrm{U}}$, is proportional to the $\sum_{m, \sigma}\left[n_{m}^{\sigma}\left(1-n_{m}^{\sigma}\right)\right]$ and $U$, the larger bias towards HS predicted for strong-field ligands can be mitigated by adopting a value of $U$ smaller than $U_{\mathrm{sc}}$. To illustrate this, we report the adiabatic energy differences computed by adopting the same $U$ value for both LS and HS for the whole series, as performed in previous studies on different compounds. ${ }^{35,36,38}$ Geometries have been optimized in each case. The computed $\Delta E_{\mathrm{H}-\mathrm{L}}$ are shown in Figure 4 for PBE and values of $U=[2,4,6,8] \mathrm{eV}$ (and Table S5). Additionally, we report $\Delta E_{\mathrm{H}-\mathrm{L}}$ computed using the average $U$ value between the linear-response $U$ computed for HS and LS, for each case. This procedure allows us to assess the validity of an approach where no bias is imposed by the use of different $U$-values while keeping an ab initio-derived parametrization of the Hubbard term. The difference $\Delta E_{\mathrm{U}, \mathrm{H}-\mathrm{L}}$ reported in the lower panel of Figure 4 shows, as expected, a lower bias towards HS when small values of $U$ are used. Thus, for weak-field ligands $U=4 \mathrm{eV}$ yields the best agreement with CASPT2/CC, with a MAE of 0.300 $\mathrm{eV}$ (Table 1), in agreement with previous studies adopting the same value of $U$ also for weak-field molecules. ${ }^{38-40}$ The strong-ligand field complexes exhibit the best agreement with reference data for $U=2 \mathrm{eV}$ with a MAE of $0.354 \mathrm{eV}$ (Table 1). Overall, the best performer is $U=2 \mathrm{eV}$ with a MAE of $0.365 \mathrm{eV}$. These results are consistent with $U$ values recently obtained by mapping $\mathrm{DFT}+\mathrm{U} \Delta E_{\mathrm{H}-\mathrm{L}}$ onto (experimentally-extracted) adiabatic energy differences and ranging between 2 and $3 \mathrm{eV}$ for molecular crystals with intermediate ligand field-strengths. ${ }^{44}$ The average- $U$ calculations yield energies differences largely deviating from the reference and consistent with $U$ values between 7 and $8 \mathrm{eV}$, thus showing the same qualitative result as the linear response- $U$ for each spin state.

Because the analysis of the density (shown in Figure 2) reveals a reduction of the deviation from the exact-density behavior upon $U$-correction, ${ }^{35,60}$ we employ again molecular orbital theory to rationalize the changes in electron density betweed DFT and DFT $+\mathrm{U}$ for the six molecular complexes. Besides, a better description of electronic correlations has been shown to affect the metal/ligand charge transfer. ${ }^{57}$ By applying $U$, a ligand $\leftrightarrow$ metal charge transfer is observed
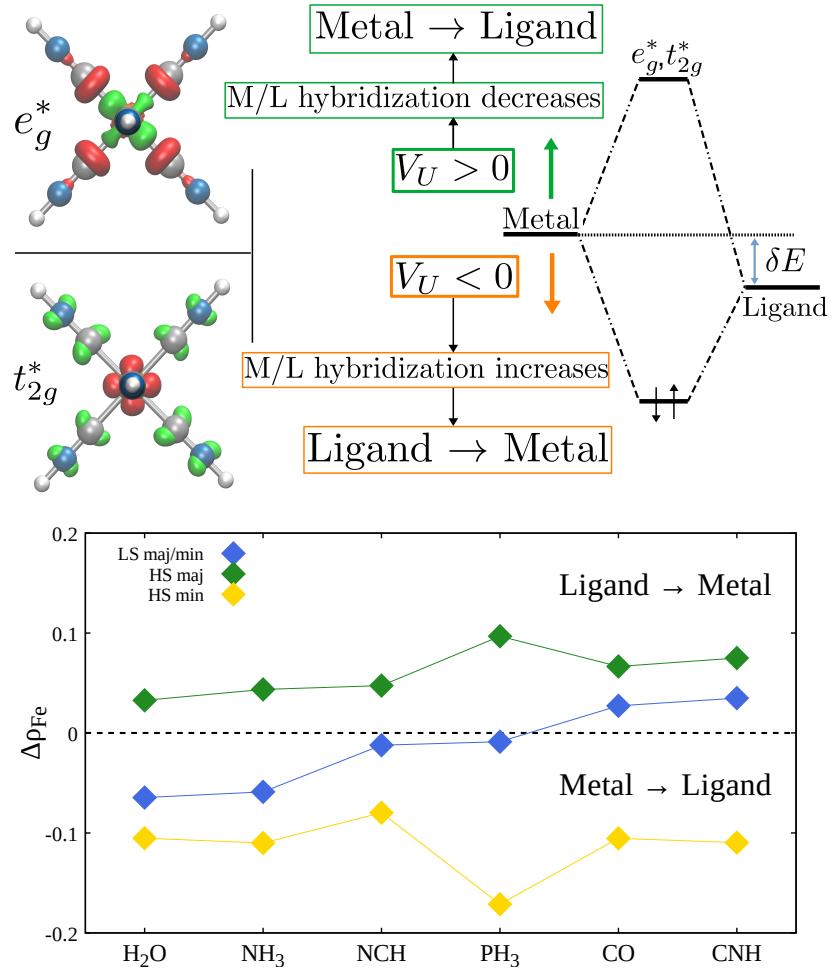

Figure 5. Molecular orbital diagram illustrating the change in $\mathrm{M} / \mathrm{L}$ hybridization upon $U$ correction and difference-plot of the $e_{g}^{*}$ and $t_{2 g}^{*}$ molecular orbitals between $U=0$ and $U_{\mathrm{sc}}$ for LS (upper panel) (red and green correspond to positive and negative isovalues). L $\rightarrow \mathrm{M}$ charge transfer computed from the Löwdin charges for the six molecular complexes using $\mathrm{PBE}+\mathrm{U}_{\mathrm{sc}}$ (lower panel).

whose sign and extent depends on both the electronic occupation and the ligand-field strength. To illustrate this, the charge transfer computed using Löwdin charges for both LS and HS are reported in Figure 5. For clarity, this analysis is performed without geometrical optimization when $U_{\mathrm{sc}}$ is employed. When the Hubbard potential is repulsive (i.e. for $n_{m}<1 / 2$ ) the corresponding KS states are pushed up in energy thus effectively decreasing the $\mathrm{M} / \mathrm{L}$ hybridization. In the case of LS, this happens when the KS states are projected onto the Fe atomic basis of $\sigma$ symmetry with respect to the ligand, i.e. the $\mathrm{d}_{\mathrm{x}^{2}-\mathrm{y}^{2}}$ and $\mathrm{d}_{\mathrm{z}^{2}}$ sets (for axes aligned along the ligands). It is crucial to recall that in this case both the occupied bonding $e_{g}$ and the unoccupied antibonding $e_{g}^{*}$ KS states feel a repulsive $V_{U}$ potential (they are shifted up in energy) whose extent depends on the projections. The d-character increases for the unoccupied $e_{g}^{*}$ and decreases for the occupied $e_{g}$ molecular orbitals thus resulting in a more ionic-like character of the metal-ligand $\sigma$-bond and in a metal $\rightarrow$ ligand charge transfer (upper panel of Figure 5). This can be seen as an upshift of the metal states with respect to the ligand's, resulting in a larger relative energy difference between these (i.e. $\delta \mathrm{E}$, blue arrow in Figure 5), and, thus, in a smaller $\mathrm{M} / \mathrm{L}$ hybridization. ${ }^{68}$

Conversely, in LS the Hubbard potential is attractive for KS states that project onto the Fe atomic basis $\left(d_{x y}, d_{x z}\right.$ and $\left.\mathrm{d}_{\mathrm{yz}}\right)$. These are molecular orbitals with $\pi$ symmetry, i.e. the $t_{2 g}$ set $\left(t_{2 g}, t_{2 g}\right.$-n and $\left.t_{2 g}^{*}\right)$, and the corresponding $n_{m}>1 / 2$ because the unoccupied $t_{2 g}^{*}$ carry a small contribution from the metal atom, as illustrated in Figure 3 . The whole $t_{2 g}$ set 
is pushed down in energy yielding an increased hybridization with the $\pi$ of the ligand and a decreased hybridization with the $\pi^{*}$ (see illustration in the upper panel of Figure 5 and left panel of Figure 3). Since the corresponding $t_{2 g}^{*}$ are unoccupied, this results in a ligand $\rightarrow$ metal charge transfer. Thus, the $e_{g}^{*}$ and $t_{2 g}^{*}$ sets respectively increase and decrease their d-character upon $U$ correction as illustrated in Figure 5 (Figures S16-S17). As the ligand-field strength increases, both effects increase in magnitude with the $\mathrm{L} \rightarrow \mathrm{M}$ charge transfer becoming larger than the $\mathrm{M} \rightarrow \mathrm{L}$, due to bigger change, upon application of $U$, in hybridization of the $t_{2 g}$ set compared to the $e_{g}$ one (Figures S4-S15). This is demonstrated by the change in occupation numbers $\left\{n_{m}^{\sigma}\right\}$ upon $U$, shown in Tables S12-S13. The M $\leftrightarrow \mathrm{L}$ charge transfer computed for LS thus changes its sign along the molecular series (Figure 5).

For HS, the whole set of occupations $\left\{n_{m}^{\sigma}\right\}>1 / 2$ and the Hubbard potential is attractive for the whole majority spin manifold resulting in a $\mathrm{L} \rightarrow \mathrm{M}$ charge transfer throughout the molecular series. For the minority spin channel, only one $n_{m}>1 / 2$ and overall the charge transfer is $\mathrm{M} \rightarrow \mathrm{L}$.

In conclusion, we show and explain the performance of $\mathrm{DFT}+\mathrm{U}_{\mathrm{sc}}$ approach in describing spin-state energetics. For weak-field molecules, such as $\left[\mathrm{Fe}\left(\mathrm{H}_{2} \mathrm{O}\right)_{6}\right]^{2+}$, the overstabilization of LS by standard PBE or LDA functionals may cancel out with the the bias introduced by the Hubbard correction thus yielding to a good agreement with the reference values. For stronger ligand-fields, the larger bias towards HS state yields to deviations from the reference CASPT2/CC values by up to several $\mathrm{eV}$. As a semiempirical approach, lower values of $U$ may be adopted to mitigate this bias, thus explaining why $U=4 \mathrm{eV}$, often employed in the literature, may perform better. Based on the analysis provided here, we anticipate a reduced energy-bias for spin-state energy differences involving a reduced change in the summation term upon spin transition. This is the case for weak-field ligandmolecules, as shown here, and when the lowest number of molecular orbitals is involved in the change of occupation upon spin transition, for example two molecular orbitals ${ }^{35,36}$ rather than four as in the present case. Finally, we rationalize the density change upon application of $U$ in terms of change in metal/ligand hybridization. This analysis offers a general tool to interprete the $U$-induced charge redistribution and allows for the explaination of previous results. ${ }^{71}$

Acknowledgement This work was funded by the French National Agency for Research (ANR-15-CE06-0003-01). Calculations were performed using resources granted by GENCI under the CINES grant number A0020907211. The froggy platform of the CIMENT infrastructure was also employed for the calculations. B.V. was supported by the Department of Energy, Basic Energy Sciences (DESC0019463). Some of the computations supporting this project were performed on High Performance Computing systems at the University of South Dakota, funded by NSF Award 1626516. Also resources of the National Energy Research Scientific Computing Center (NERSC), a U.S. Department of Energy Office of Science User Facility operated under Contract No. DE-AC02-05CH11231, were used for this project.

Supporting Information Available: Computational details, CASPT2 $\Delta E_{\mathrm{H}-\mathrm{L}}$, CASPT2-optimized molecular orbitals, computed summation $\sum_{m, \sigma}\left[n_{m}^{\sigma}\left(1-n_{m}^{\sigma}\right)\right]$ for LDA, eigenvalues of occupation matrix, projected density of states, Hirshfeld population analysis, cartesian coordinates of the optimized geome- tries. This material is available free of charge via the Internet at http://pubs.acs.org/.

\section{References}

(1) Wilbraham, L.; Verma, P.; Truhlar, D. G.; Gagliardi, L. Ciofini, I. Multiconfiguration Pair-Density Functional Theory Predicts Spin-State Ordering in Iron Complexes with the same Accuracy as Complete Active Space Second-Order Perturbation Theory at a Significantly Reduced Computational Cost. J. Phys. Chem. Lett. 2017, 8, 2026-2030.

(2) Domingo, A.; Àngels Carvajal, M.; De Graaf, C. Spin Crossover in $\mathrm{Fe}(\mathrm{II})$ Complexes: An Ab Initio Study of Ligand $\sigma$-Donation. Int. J. Quantum Chem. 2010, 110, 331-337.

(3) Radoń, M. Benchmarking Quantum Chemistry Methods for Spin-State Energetics of Iron Complexes Against Quantitative Experimental Data. Phys. Chem. Chem. Phys. 2019, 21, 48544870.

(4) Cirera, J.; Paesani, F. Theoretical Prediction of Spin-Crossover Temperatures in Ligand-Driven Light-Induced Spin Change Systems. Inorg. Chem. 2012, 51, 8194-8201.

(5) Pierloot, K.; Vancoillie, S. Relative Energy of the High- $\left({ }^{5} T_{2 g}\right)$ and Low- $\left({ }^{1} A_{1 g}\right)$ Spin States of $\left[\mathrm{Fe}\left(\mathrm{H}_{2} \mathrm{O}\right)^{6}\right]^{2+},\left[\mathrm{Fe}\left(\mathrm{NH}_{3}\right)^{6}\right]^{2+}$ and $\left[\mathrm{Fe}(\mathrm{bpy})_{3}\right]^{2+}:$ CASPT2 Versus Density Functional Theory. J. Chem. Phys. 2006, 125, 124303.

(6) Swart, M. Accurate Spin-State Energies for Iron Complexes. J. Chem. Theory Comput. 2008, 4, 2057-2066.

(7) Droghetti, A.; Alfè, D.; Sanvito, S. Assessment of Density Functional Theory for Iron(II) Molecules Across the Spin-Crossover Transition. J. Chem. Phys. 2012, 137, 124303.

(8) Cirera, J.; Via-Nadal, M.; Ruiz, E. Benchmarking Density Functional Methods for Calculation of State Energies of first Row Spin-Crossover Molecules. Inorg. Chem. 2018, 57, 14097 14105.

(9) Kumar, K. S.; Ruben, M. Emerging Trends in Spin Crossover (SCO) Based Functional Materials and Devices. Coordin Chem. Rev. 2017, 346, 176-205.

(10) Molnár, G.; Mikolasek, M.; Ridier, K.; Fahs, A.; Nicolazzi, W.; Bousseksou, A. Molecular Spin Crossover Materials: Review of the Lattice Dynamical Properties. Ann. Phys. 2019, 531 1900076.

(11) Resines-Urien, E.; Burzurí, E.; Fernandez-Bartolome, E.; García García-Tuñón, M. A.; de la Presa, P.; Poloni, R.; Teat, S. J.; Sánchez-Costa, J. A switchable iron-based coordination polymer toward reversible acetonitrile electro-optical readout. Chem. Sci. 2019, 10, 6612-6616.

(12) Resines-Urien, E.; Piñeiro López, L.; Fernandez-Bartolome, E.; Gamonal, A.; Garcia-Hernandez, M.; Sánchez Costa, J. Covalent post-synthetic modification of switchable iron-based coordination polymers by volatile organic compounds: a versatile strategy for selective sensor development. Dalton Trans. 2020, 49, 7315-7318.

(13) Swart, M.; Groenhof, A. R.; Ehlers, A. W.; Lammertsma, K Validation of Exchange-Correlation Functionals for Spin States of Iron Complexes. J. Phys. Chem. A 2004, 108, 5479-5483.

(14) Droghetti, A.; Alfè, D.; Sanvito, S. Assessment of Density Functional Theory for Iron(II) Molecules Across the Spin-Crossover Transition. J. Chem. Phys. 2012, 137, 124303.

(15) Song, S.; Kim, M.-C.; Sim, E.; Benali, A.; Heinonen, O.; Burke, K. Benchmarks and Reliable DFT Results for Spin Gaps of Small Ligand $\mathrm{Fe}(\mathrm{II})$ Complexes. J. Chem. Theory Comput. 2018, 14, 2304-2311.

(16) Fouqueau, A.; Casida, M. E.; Daku, L. M. L.; Hauser, A. Neese, F. Comparison of Density Functionals for Energy and Structural Differences Between the High- $\left.-{ }^{5} T_{2 g}:\left(t_{2 g}\right)^{4}\left(e_{g}\right)^{2}\right]$ and Low- $\left[{ }^{1} A_{1 g}:\left(t_{2 g}\right)^{6}\left(e_{g}\right)^{0}\right]$ Spin States of Iron(II) Coordination Compounds. II. More Functionals and the Hexaminoferrous Cation, $\left[\mathrm{Fe}\left(\mathrm{NH}_{3}\right)^{6}\right]^{2+}$. J. Chem. Phys. 2005, 122, 044110

(17) Radoń, M. Revisiting the Role of Exact Exchange in DFT SpinState Energetics of Transition Metal Complexes. Phys. Chem. Chem. Phys. 2014, 16, 14479-14488.

(18) Mortensen, S. R.; Kepp, K. P. Spin Propensities of Octahedral Complexes from Density Functional Theory. J. Phys. Chem. A 2015, 119, 4041-4050.

(19) Ioannidis, E. I.; Kulik, H. J. Towards Quantifying the Role of Exact Exchange in Predictions of Transition Metal Complex Properties. J. Chem. Phys. 2015, 143, 034104.

(20) Kulik, H. J. Perspective: Treating Electron Over-Delocalization with the DFT $+U$ Method. J. Chem. Phys. 2015, 142, 240901.

(21) Rehier, M.; Salomon, O.; Arthus Hess, B. Reparameterization of Hybrid Functionals Based on Energy Differences of States of Different Multiplicit. Theor. Chem. Acc. 2001, 107, 48-55.

(22) Prokopiou, G.; Kronik, L. Spin-State Energetics of Fe Complexes from an Optimally Tuned Range-Separated Hybrid Functional. Chem.-Eur. J. 2018, 24, 5173-5182.

(23) Anisimov, V. I.; Zaanen, J.; Andersen, O. K. Band Theory and Mott Insulators: Hubbard $U$ Instead of Stoner I. Phys. Rev. B 1991, 44, 943.

(24) Anisimov, V. I.; Solovyev, I.; Korotin, M.; Czyżyk, M.; Sawatzky, G. Density-Functional Theory and NiO Photoemission Spectra. Phys. Rev. B 1993, 48, 16929. 
(25) Czyżyk, M.; Sawatzky, G. Local-Density Functional and OnSite Correlations: The Electronic Structure of $\mathrm{La}_{2} \mathrm{CuO}_{4}$ and $\mathrm{LaCuO}_{3}$. Phys. Rev. B 1994, 49, 14211.

(26) Solovyev, I.; Dederichs, P.; Anisimov, V. Corrected atomic limit in the local-density approximation and the electronic structure of d impurities in Rb. Phys. Rev. B 1994, 50, 16861.

(27) Perdew, J. P.; Zunger, A. Self-Interaction Correction to DensityFunctional Approximations for Many-Electron Systems. Phys. Rev. B 1981, 23, 5048 .

(28) Perdew, J. P.; Parr, R. G.; Levy, M.; Balduz Jr, J. L. DensityFunctional Theory for Fractional Particle Number: Derivative Discontinuities of the Energy. Phys. Rev, Lett, 1982, 49, 1691.

(29) Perdew, J. P.; Levy, M. Physical Content of the Exact KohnSham Orbital Energies: Band Gaps and Derivative Discontinuities. Phys. Rev. Lett. 1983, 51, 1884.

(30) Zhang, Y.; Yang, W. A Challenge for Density Functionals: SelfInteraction Error Increases for Systems with a Noninteger Number of Electrons. J. Chem. Phys. 1998, 109, 2604-2608.

(31) Cramer, C. J.; Truhlar, D. G. Density Functional Theory for Transition Metals and Transition Metal Chemistry. Phys. Chem. Chem. Phys. 2009, 11, 10757-10816.

(32) Himmetoglu, B.; Floris, A.; de Gironcoli, S.; Cococcioni, M. Hubbard-Corrected DFT Energy Functionals: The LDA + U Description of Correlated Systems. Int. J. Quantum Chem. 2014 114, 14-49.

(33) Poloni, R.; Mariano, A. L.; Prendergast, D.; GarciaBarriocanal, J. Probing the electric field-induced doping mechanism in $\mathrm{YBa}_{2} \mathrm{Cu}_{3} \mathrm{O}_{7}$ using computed $\mathrm{Cu} \mathrm{K}$-edge x-ray absorption spectra. The Journal of Chemical Physics 2018, 149, 234706 .

(34) Tolba, S. A.; Gameel, K. M.; Ali, B. A.; Almossalami, H. A.; Allam, N. K. In Density Functional Calculations; Yang, G. Ed.; IntechOpen: Rijeka, 2018; Chapter 1.

(35) Kulik, H. J.; Cococcioni, M.; Scherlis, D. A.; Marzari, N. Density Functional Theory in Transition-Metal Chemistry: A SelfConsistent Hubbard $U$ Approach. Phys. Rev. Lett. 2006, 97 103001.

(36) Kulik, H. J.; Marzari, N. Systematic Study of FirstRow Transition-Metal Diatomic Molecules: A Self-Consistent DFT $+U$ Approach. J. Chem. Phys. 2010, 133, 114103.

(37) Hsu, H.; Blaha, P.; Cococcioni, M.; Wentzcovitch, R. M. SpinState Crossover and Hyperfine Interactions of Ferric Iron in $\mathrm{MgSiO}_{3}$ Perovskite, Phys, Rev, Lett, 2011, 106, 118501.

(38) Scherlis, D. A.; Cococcioni, M.; Sit, P.; Marzari, N. Simulation of Heme Using DFT $+\mathrm{U}$ : A Step toward Accurate Spin-State Energetics. J. Phys. Chem. B 2007, 111, 7384-7391, PMID 17547444

(39) Zhang, Y.; Jiang, H. Intra-and Interatomic Spin Interaction by the Density Functional Theory plus $U$ Approach: A Critical Assessment. J. Chem. Theory Comput. 2011, 7, 2795-2803.

(40) Ali, M. E.; Sanyal, B.; Oppeneer, P. M. Electronic Structure, Spin-States, and Spin-Crossover Reaction of Heme-Related Fe-Porphyrins: A Theoretical Perspective. J. Phys. Chem. B 2012, 116, 5849-5859, PMID: 22512398.

(41) Lebègue, S.; Pillet, S.; Ángyán, J. G. Modeling Spin-Crossover Compounds by Periodic DFT $+\mathrm{U}$ Approach. Phys. Rev. B 2008, 78, 024433.

(42) Panchmatia, P. M.; Ali, M. E.; Sanyal, B.; Oppeneer, P. M. Halide Ligated Iron Porphines: A DFT + U and UB3LYP Study. J. Phys. Chem. A 2010, 114, 13381-13387, PMID: 21126064.

(43) Maldonado, P.; Kanungo, S.; Saha-Dasgupta, T.; Oppeneer, P. M. Two-Step Spin-Switchable Tetranuclear Fe(II) Molecular Solid: Ab Initio Theory and Predictions. Phys. Rev. $B$ 2013, 88, 020408 .

(44) Vela, S.; Fumanal, M.; Cirera, J.; Ribas-Arino, J. Thermal Spin Crossover in $\mathrm{Fe}(\mathrm{ii})$ and $\mathrm{Fe}(\mathrm{iii})$. Accurate Spin State Energetics at the Solid State. Phys. Chem. Chem. Phys. 2020, 22, 49384945.

(45) Cococcioni, M.; De Gironcoli, S. Linear Response Approach to the Calculation of the Effective Interaction Parameters in the LDA $+U$ Method. Phys. Rev. B 2005, 71, 035105

(46) Pierloot, K.; Phung, Q. M.; Domingo, A. Spin State Energetics in First-Row Transition Metal Complexes: Contribution of (3s3p) Correlation and its Description by Second-Order Perturbation Theory. J. Chem. Theory Comput. 2017, 13, 537-553.

(47) Phung, Q. M.; Feldt, M.; Harvey, J. N.; Pierloot, K. Toward highly Accurate Spin State Energetics in First-Row Transition Metal Complexes: A Combined CASPT2/CC Approach. J. Chem. Theory Comput. 2018, 14, 2446-2455.

(48) Hsu, H.; Umemoto, K.; Cococcioni, M.; Wentzcovitch, R. FirstPrinciples Study for Low-Spin $\mathrm{LaCoO}_{3}$ with a Structurally Consistent Hubbard U. Phys. Rev. B 2009, 79, 125124

(49) Giannozzi, P. et al. QUANTUM ESPRESSO: a modular and open-source software project for quantum simulations of materials. J. Phys: Condens. Matter 2009, 21, 395502.

(50) Shiozaki, T. BAGEL: Brilliantly Advanced General ElectronicStructure Library Modular and Open-Source Software Project for Quantum Simulations of Materials. WIREs Comput. Mol. Sci 2018, 8:e1331.

(51) http://www.nubakery.org under the GNU General Public License.

(52) Tao, J.; Perdew, J. P.; Staroverov, V. N.; Scuseria, G. E. Climbing the Density Functional Ladder: Nonempirical MetaGeneralized Gradient Approximation Designed for Molecules and Solids. Phys. Rev. Lett. 2003, 91, 146401.

(53) Staroverov, V. N. Scuseria, G. E. Tao, J.; Perdew, J. P. Comparative Assessment of a New Nonempirical Density Functional: Molecules and Hydrogen-Bonded Complexes. J. Chem. Phys. 2003, 119, 12129-12137.

(54) Ghigo, G.; Roos, B. O.; ÃEke Malmqvist, P. A modified definition of the zeroth-order Hamiltonian in multiconfigurational perturbation theory (CASPT2). Chemical Physics Letters 2004, 396, $142-149$.

(55) Neese, F. The ORCA Program System. WIREs Comput. Mol. Sci. 2012, 2, 73-78.

(56) Neese, F. Software Update: The ORCA Program System, Version 4.0. WIREs Comput. Mol. Sci. 2018, 8, e1327.

(57) Fumanal, M.; Wagner, L. K.; Sanvito, S.; Droghetti, A. Diffusion Monte Carlo Perspective on the Spin-State Energetics of $[\mathrm{Fe}(\mathrm{NCH}) 6] 2+$. Journal of Chemical Theory and Computation 2016, 12, 4233-4241, PMID: 27500854.

(58) Ganzenmüller, G.; Berkaïne, N.; Fouqueau, A.; Casida, M. E. Reiher, M. Comparison of Density Functionals for Differences Between the High- $\left({ }^{5} T_{2 g}\right)$ and Low- $\left({ }^{1} A_{1 g}\right)$ Spin States of Iron(II) Compounds. IV. Results for the Ferrous Complexes $\left[\mathrm{Fe}(\mathrm{L})\left(\mathrm{NHS}_{4}\right)\right]$. J. Chem. Phys. 2005, 122, 234321.

(59) Cohen, A. J.; Mori-Sánchez, P.; Yang, W. Challenges for Density Functional Theory. Chem. Rev. 2011, 112, 289-320.

(60) Zhao, Q.; Ioannidis, E. I.; Kulik, H. J. Global and Local Curvature in Density Functional Theory. J. Chem. Phys. 2016, 145, 054109.

(61) Sham, L. J.; Schlüter, M. Density-Functional Theory of the Energy Gap. Phys. Rev. Lett. 1983, 51, 1888.

(62) Mori-Sánchez, P.; Cohen, A. J. The Derivative Discontinuity of the Exchange-Correlation Functional. Phys. Chem. Chem. Phys. 2014, 16, 14378-14387.

(63) Stein, T.; Autschbach, J.; Govind, N.; Kronik, L.; Baer, R Curvature and Frontier Orbital Energies in Density Functional Theory. The Journal of Physical Chemistry Letters 2012, 3, 3740-3744, PMID: 26291104.

(64) Johnson, E. R.; Otero-de-la Roza, A.; Dale, S. G. Extreme Density-Driven Delocalization Error for a Model SolvatedElectron System. J. Chem. Phys. 2013, 139, 184116.

(65) Janak, J. Proof that $\frac{\partial E}{\partial n_{i}}=\varepsilon$ in Density-Functional Theory. Phys. Rev. B 1978, 18, 7165 .

(66) Janet, J. P.; Zhao, Q.; Ioannidis, E. I.; Kulik, H. J. Density functional theory for modelling large molecular adsorbate-surface interactions: a mini-review and worked example. Molecular Simulation 2017, 43, 327-345.

(67) Chen, J.; Millis, A. J.; Marianetti, C. A. Density functiona plus dynamical mean-field theory of the spin-crossover molecule $\mathrm{Fe}(\text { phen })_{2}(\mathrm{NCS})_{2}$. Phys. Rev. B 2015, 91, 24111.

(68) Jean, Y. Molecular Orbitals of Transition Metal Complexes; OUP Oxford, 2005.

(69) Huang, B. The Screened Pseudo-Charge Repulsive Potential in Perturbed Orbitals for Band Calculations by DFT $+U$. Phys. Chem. Chem. Phys. 2017, 19, 8008-8025.

(70) Linscott, E. B.; Cole, D. J.; Payne, M. C.; O'Regan, D. D. Role of Spin in the Calculation of Hubbard $U$ and Hund's $J$ Parameters from First Principles. Phys. Rev. B 2018, 98, 235157.

(71) Gani, T. Z.; Kulik, H. J. Where does the Density Localize? Convergent Behavior for Global Hybrids, Range Separation, and DFT $+U$. J. Chem. Theory Comput. 2016, 12, 5931-5945. 


\section{Graphical TOC Entry}

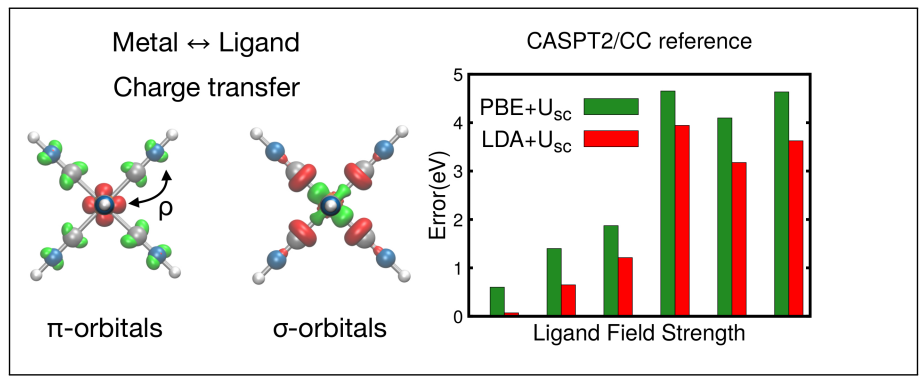

